\title{
On the applicability of numerical tools for simulating wave- ports close to the cutoff frequency
}

\author{
Eman Mohamed Eldesouki, Khalid Mustafa Ibrahim, Ahmed Mohmed Attiya \\ Microwave Engineering Department, Electronics Research Institute (ERI), Cairo, Egypt
}

\begin{tabular}{l}
\hline \hline Article Info \\
\hline Article history: \\
Received Jan 14, 2021 \\
Revised Oct 10, 2021 \\
Accepted Oct 28, 2021 \\
\hline
\end{tabular}

Keywords:

Numerical simulation

Electromagnetic CAD

Waveguide

\begin{abstract}
This paper focuses on a common drawback in electromagnetic numerical computer aided design computer aided design (CAD) tools: high frequency structure simulator (HFSS), computer simulation technology (CST) and FEKO, where the excitation by using a wave-port below and close to the cutoff frequency has unreliable values for the reflection coefficient. An example for such problem is presented in the design of a dual horn antenna fed by two different waveguide sections. To overcome this numerical error in the results of these CAD tools, a tapered waveguide section is used in the simulation as an excitation mechanism to the feeding waveguide. The cross section of the input port at this tapered waveguide section is designed to have a cutoff frequency smaller than the lowest frequency under investigation for the original problem. Then, by extracting the effect of the tapered section from the obtained reflection coefficient, it would be possible to obtain the reflection coefficient of the original problem.
\end{abstract}

This is an open access article under the CC BY-SA license.

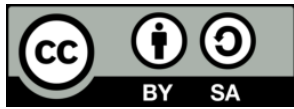

\section{Corresponding Author:}

Eman Mohamed Eldesouki

Microwave Engineering Department, Electronics Research Institute (ERI)

Joseph Tito St, Huckstep, El Nozha, Cairo Governorate, Egypt

Email:eman@eri.sci.eg

\section{INTRODUCTION}

Electromagnetic numerical simulation computer aided design (CAD) tools like high frequency structure simulator (HFSS) [1], computer simulation technology (CST) [2] and FEKO [3] have critical roles in analysis and design of different antennas and microwave circuit configurations [4]-[13], [14]-[17]. The numerical techniques of these CAD tools like finite element method, finite difference method and method of moments are well investigated in literature [18]-[20]. However, these CAD tools are not open-source codes to enable their users to add or modify any feature to the available ones. This is the motivation to study the possibility of using the available capabilities of these CAD tools in different ways to simulate some problems which may not be suitable to be simulated by using conventional direct steps. As an example of these problems is the problem of using a wave-port below or close to its cutoff frequency.

In these CAD tools, the wave-port is equivalent to a semi-infinite waveguide on the other side of the wave-port. This semi-infinite waveguide by default is excited by its dominant mode. In addition, higher order modes can also be included in simulation if they are required in the solution. From the analytical point of view, there is no wave propagation in the waveguide below its dominant cutoff frequency and subsequently the reflection coefficient at the wave-port below the cutoff frequency should be $0 \mathrm{~dB}$ [21]. However, from the results of different simulations, the authors noted that this condition is not valid for both HFSS, CST and FEKO. An example of a dual-band horn antenna [22], [23] fed by two different waveguides is presented in the Section 2 as an example on this problem. The problem of using wave-port analysis below and close to the cutoff frequency has a significant importance in simulating multi-band multi-fed antennas. It is also very 
important in simulating artificial structures like epsilon-near-zero metamaterial which can be designed by a waveguide section operating near its cutoff frequency [24], [25].

In this paper, an additional tapered waveguide section [26], [27] is used as an excitation to the original feeding waveguide. This tapered section is designed such that its feeding port would have a cutoff frequency much less than any frequency of interest in the required analysis. Thus, its wave-port is always above the cutoff in the proposed simulation. Then, this tapered section is simulated separately to extract its parameters. Finally, the parameters of the tapered section is extracted from the simulation results. By using this method, it would be possible to obtain the results for waveguide-excited structures below and close to the cutoff frequency.

\section{FORMULATION OF THE PROBLEM}

In this section we present an example of a dual-band horn antenna fed by using two different orthogonal waveguides as shown in Figure 1. This antenna is designed to be operating in $\mathrm{K} / \mathrm{Ka}$ bands from 20 to $22 \mathrm{GHz}$ and from 33 to $35 \mathrm{GHz}$ respectively. The length of the conical structure $L_{1}=35 \mathrm{~mm}$, the radius of the radiating aperture is $r=16.637 \mathrm{~mm}$, and the radius of the lower feeding aperture is $r_{o}=5 \mathrm{~mm}$. This conical structure is connected to a short circuited cylindrical waveguide section of length $L_{2}=12 \mathrm{~mm}$ and a radius $r_{o}=5 \mathrm{~mm}$, respectively. The antenna is fed from this cylindrical waveguide section by using two orthogonal rectangular waveguide sections as shown in Figure 1. The waveguide section waveguide 1 (WG1) has a cross section dimensions $9 \times 1.675 \mathrm{~mm}$. These dimensions have a cutoff frequency for the dominant TE10 mode at $16.65 \mathrm{GHz}$. On the other hand, the waveguide section WG2 has a cross section dimensions $6.228 \times 1.87 \mathrm{~mm}$. These dimensions have a cutoff frequency for the dominant TE10 mode at 24.2 GHz.

This antenna configuration is simulated by using both HFSS and CST simulation tools. Two waveports are used as excitations for the feeding rectangular waveguide sections. Figures 2 and 3 show the simulation results for the scattering parameter of this antenna configuration at the required two frequency bands by using both HFSS and CST. It can be noted that the performance of the WG1 which is designed to be the feeding waveguide section for the lower frequency band has normal conditions at lower band. In this case the reflection coefficient of WG1; $S_{11}$; is less than $10 \mathrm{~dB}$ in the operating $\mathrm{K}$ band from 20 to $22 \mathrm{GHz}$ as shown in Figure 2(a). On the other hand, it has a negligible transmission to WG2; $S_{21}$; as shown in Figure 3(a), because the second waveguide section is blow the cutoff in this frequency band. The unconventional result in this case is that the reflection coefficient of WG2, which is below cutoff frequency at the lower band, has a very small reflection coefficient as shown in Figure 2(a).

This notice introduces a question: If the excitation power from the wave-port 2 connected WG2 is not reflected and not transferred to port 1, where this power is dissipated? For such simple antenna configuration, it quite clear that the WG2 is below the cutoff frequency at the lower frequency band and the expected reflection coefficient should be $0 \mathrm{~dB}$. At quite close to the cutoff frequency of WG2, the problem may need more justification and the reflection coefficient may not be exactly $0 \mathrm{~dB}$. This was the motivation here to find a method to overcome this inconsistence.

On the other hand, the simulation results of the upper frequency band, where both feeding waveguides are above cutoff, are consistent for both the reflection and transmission coefficients as shown in Figure 2(b) and Figure 3(b). The only note on the results of this upper frequency band is the high coupling between the two feeding ports. This is explained due to that both waveguides are above cutoff frequency in this case. This problem is solved for this antenna configuration by adding a low pass filter to WG1 to decouple the two feeding waveguides at the upper frequency band. This is a design problem, not a simulation problem.

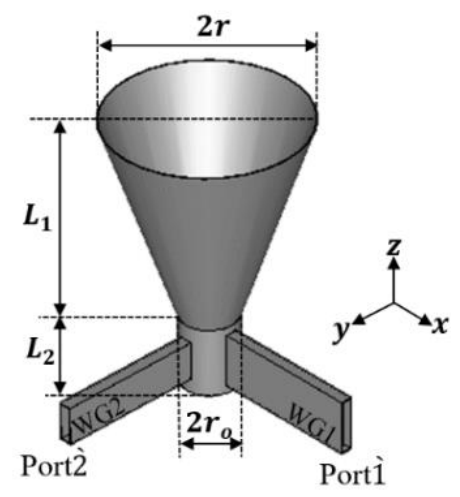

Figure 1. Geometry of a dual-band conical horn antenna fed by two different waveguides 


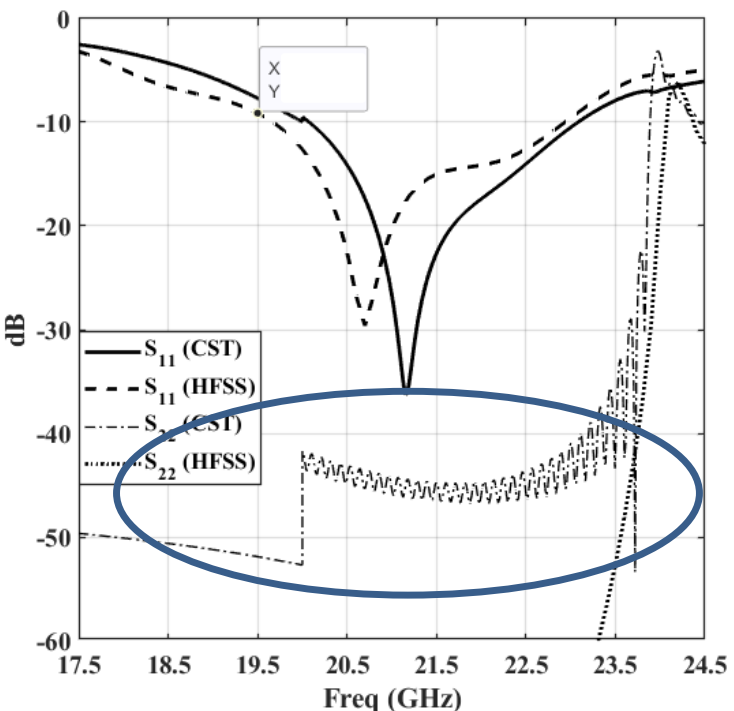

(a)

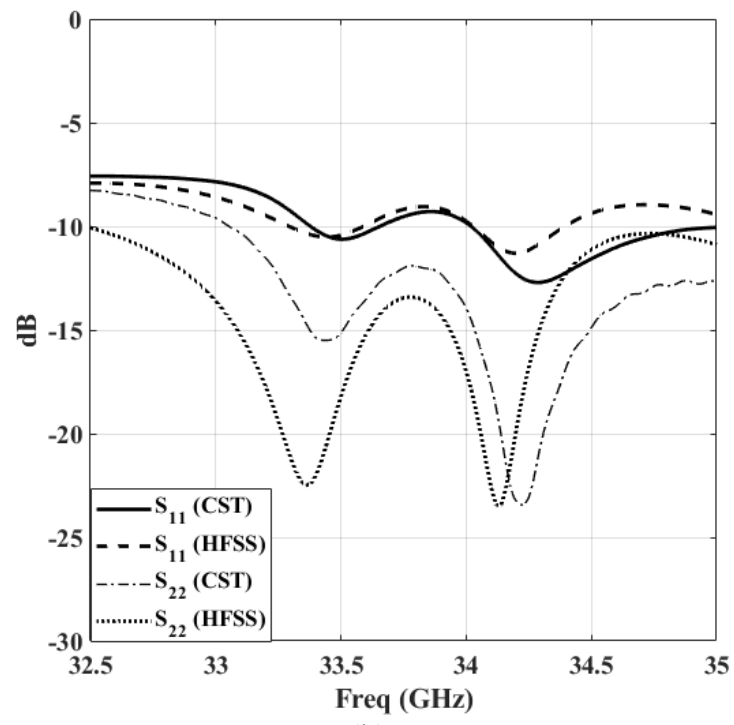

(b)

Figure 2. Simulated reflection coefficient of the conical horn antenna feed by two rectangular waveguide ports (a) lower frequency band, (b) upper frequency band

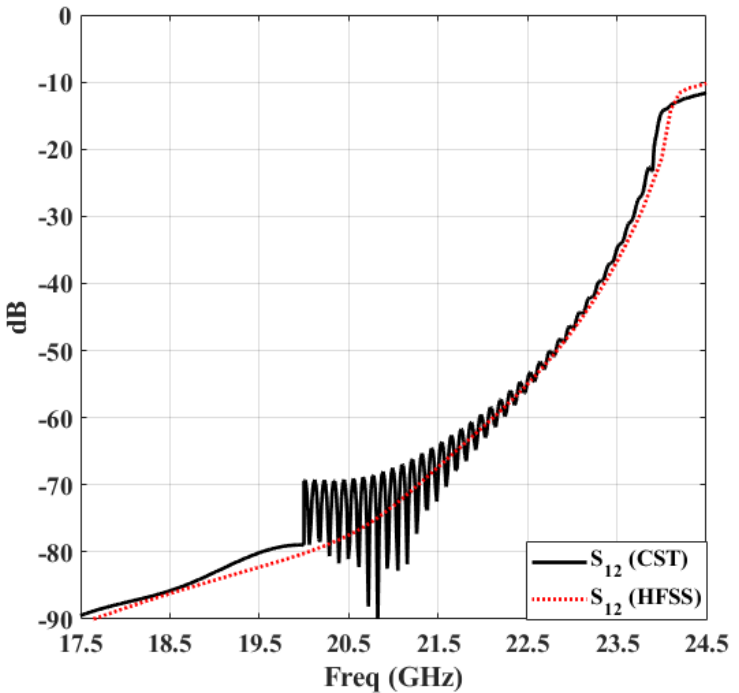

(a)

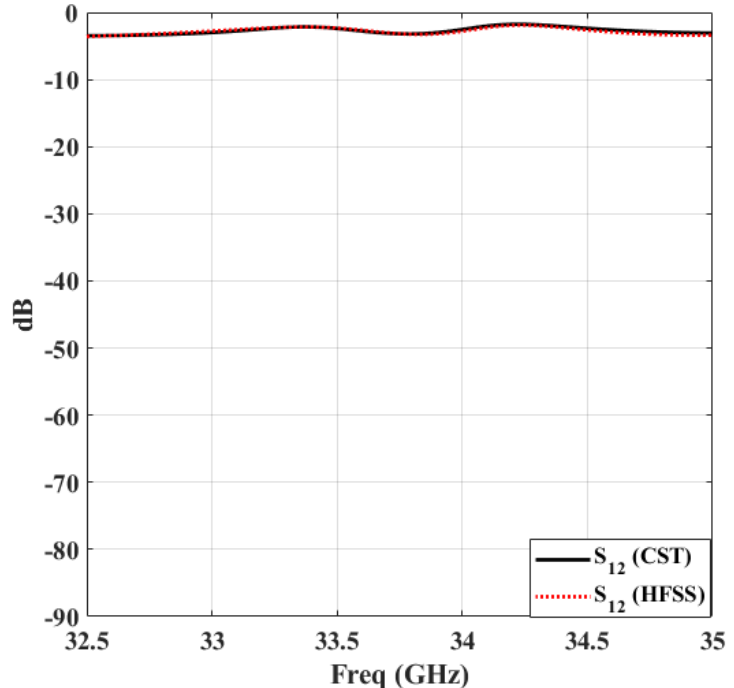

(b)

Figure 3. Simulated transmission coefficient of the conical horn antenna feed by two rectangular waveguide ports (a) lower frequency band and (b) upper frequency band

As another check to this wave-port problem, we studied the scattering parameters of a straight waveguide section of the dimensions of WG2 with a length of $100 \mathrm{~mm}$. The walls of the waveguide is assumed to be perfect electric conductor and the filling dielectric is vacuum to avoid any losses in the waveguide section. Figure 4 shows the reflection and transmission coefficients for this configuration. In addition to HFSS and CST, we used also FEKO in this simulation. It can be noted that the results of the transmission coefficient below the cutoff frequency, $f_{c}=24.2 \mathrm{GHz}$, is consistent with the expected performance where it has a very small value. However, the reflection coefficient below the cutoff frequency should be around $0 \mathrm{~dB}$ which is not the case as shown in Figure 4(a). This result proves that the inconsistence in the simulation of the above dual-band horn antenna is due to the excitation of the feeding waveguide and is not related to the antenna configuration. On the other hand, the transmission coefficient is consistent below and above the cutoff frequency as shown in Figure 4(b). 


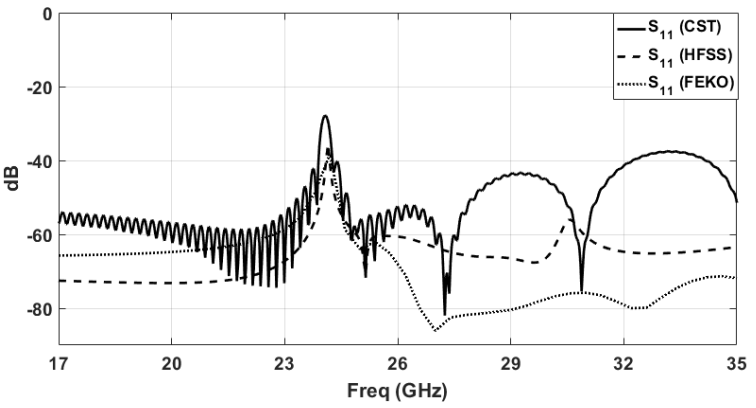

(a)

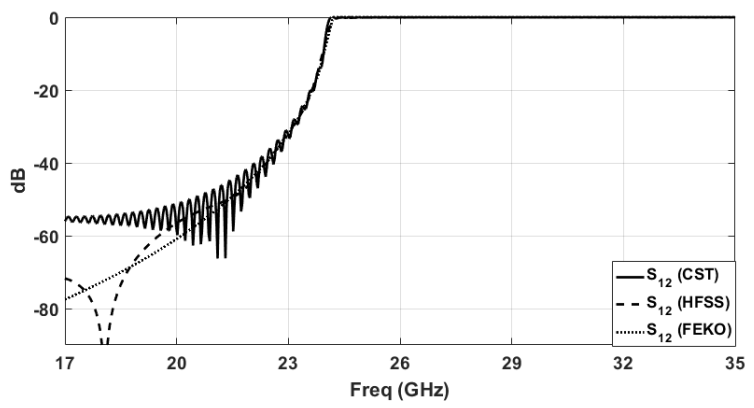

(b)

Figure 4. S parameter of a straight waveguide section WG2 with dimensions $6.228 \times 1.87 \times 100 \mathrm{~mm}$, (a) reflection coefficient and (b) transmission coefficient

\section{TAPERED WAVEGUIDE FEEDING STRUCTURE}

Based on the above results of the antenna configuration and the straight waveguide section, it can be concluded that the conventional wave-port model is not an adequate model below and close to its cutoff frequency. Thus, it would be required to use a wave-port with larger dimensions to have a cutoff frequency much lower than the lowest frequency of interest in the required analysis. This larger wave-port is not suitable to be used directly on the smaller aperture of the feeding waveguide. Thus, a tapered waveguide section which have an end with the larger dimensions and the other end with the dimensions of the cross section of the original feeding waveguide would be required to connect the larger wave port to the feeding waveguide. Figure 5 shows the geometry of the proposed tapered section. The tapering is assumed to be linear for simplicity. However, other configurations like exponential taper may have a better performance. The cross section at port 1 is assumed to be the same as WG2, $6.228 \times 1.87 \mathrm{~mm}$. On the other hand, the cross section at port 2 would have dimensions $18 \times 1.87 \mathrm{~mm}$. In this case the cutoff frequency of port 2 would be nearly one third the corresponding cutoff frequency of port 1 . The length of the tapered section $L_{t}$ is $40 \mathrm{~mm}$.

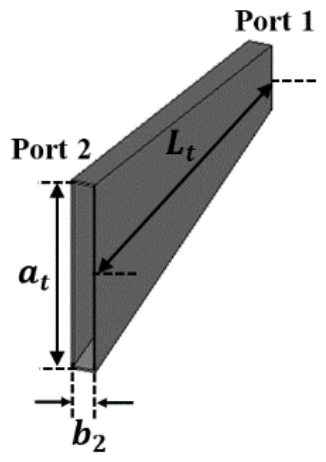

Figure 5. Geometry of tapered waveguide section

To obtain the characteristics of this tapered section, two identical tapered sections are connected through their ports 1 and $1^{\prime}$ as shown in Figure 6 . Then the excitation wave ports would be applied on the outer ports 2 and $2^{\prime}$. In this case the excitation wave ports have cutoff frequency around $8 \mathrm{GHz}$ while the required analysis is above $17 \mathrm{GHz}$. Figure 7 shows the obtained reflection and transmission coefficients. It can be noted that the full transmission will be obtained above the cutoff frequency of port 1 which is $24.2 \mathrm{GHz}$. Also, below the cutoff frequency of port 1 , the reflection coefficient is nearly $0 \mathrm{~dB}$ which is consistent with the normal performance. To extract the parameters of a single tapered section, the S-parameters of the two connected tapered sections are converted to ABCD matrix form [11]. Since both tapered sections are identical, the elements of the $\mathrm{ABCD}_{\mathrm{T}}$ matrix of a single tapered section can be extracted from the ABCD matrix of the two connected tapered section by solving the (1):

$$
\left[\begin{array}{ll}
A_{T} & B_{T} \\
C_{T} & D_{T}
\end{array}\right]\left[\begin{array}{ll}
D_{T} & B_{T} \\
C_{T} & A_{T}
\end{array}\right]=\left[\begin{array}{ll}
A & B \\
C & D
\end{array}\right]
$$




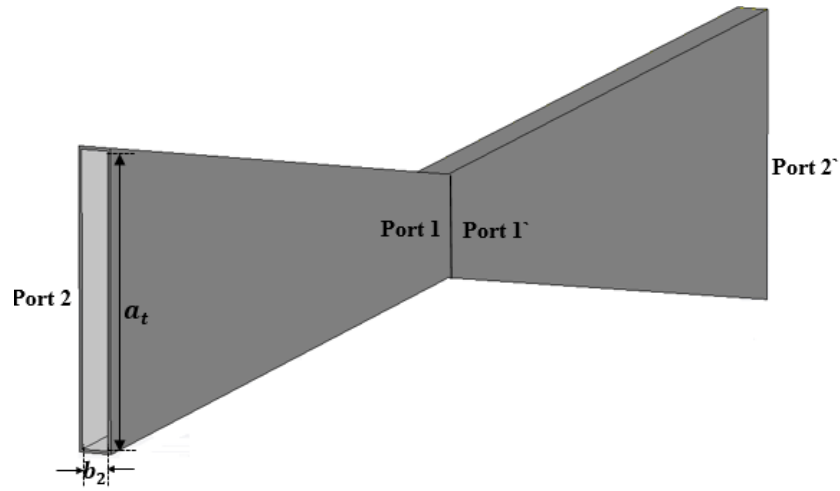

Figure 6. Geometry of two connected tapered sections

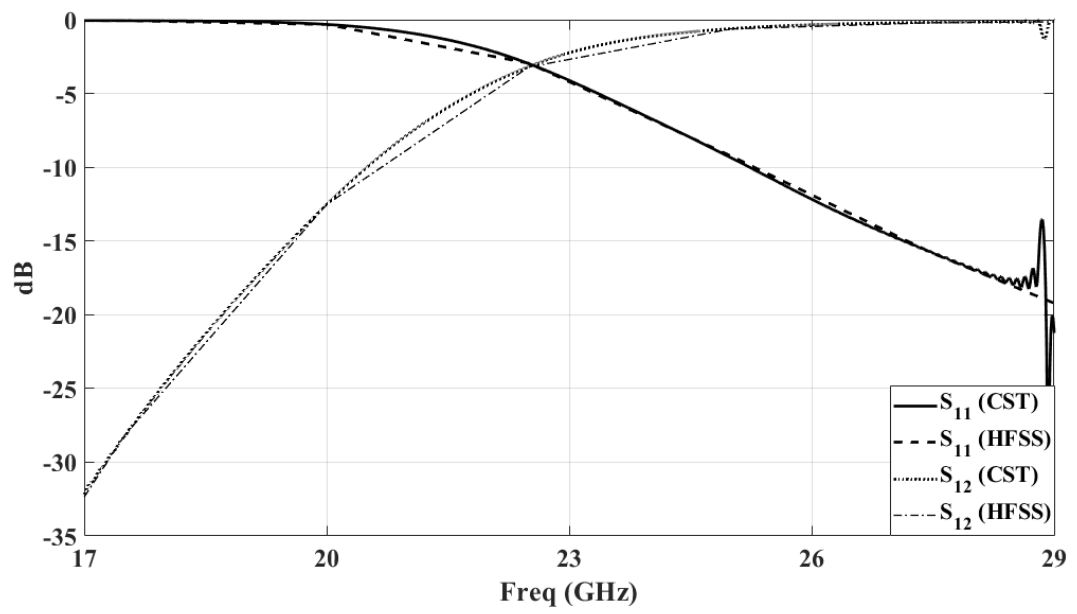

Figure 7. Reflection and transmission coefficients of two connected tapered sections

\section{APPLICATION OF TAPERED WAVEGUIDE SECTION}

In this section, the proposed tapered section presented in Section 3 is added to the problem discussed in Section 2 to correct the simulation results below and close to the cutoff frequency of WG2. For the problem of the straight waveguide, two tapered sections are added to the two ends as shown in Figure 8. Figure 9 shows the resulting reflection and transmission coefficients of this configuration (in solid lines). It can be noted that below the cutoff frequency the reflection coefficient is nearly $0 \mathrm{~dB}$ as shown in Figure 9(a), while the transmission coefficient is nearly $0 \mathrm{~dB}$ above the cutoff frequency as shown in Figure 9(b). To extract the S-parameters of the waveguide section only, the obtained S-parameters of waveguide section with the two tapered sections is converted to ABCD matrix. Then by extracting the ABCD matrix of the two tapered section one can obtain the ABCD matrix of the waveguide only. Then, the ABCD matrix of the waveguide only is converted back to scattering matrix to obtain the reflection and transmission coefficients of the waveguide section only as shown in Figure 9 (in dashed lines).

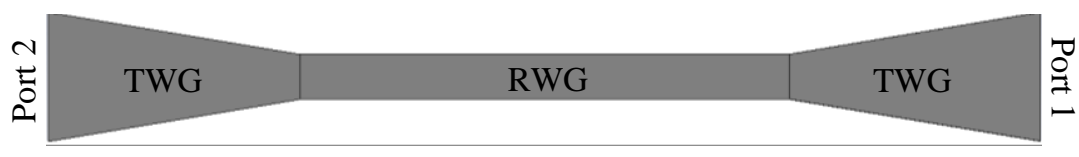

Figure 8. Geometry of a straight waveguide section with two tapered sections

This result of the straight waveguide section shows the advantage of using the tapered section for the excitation to correct the simulation results below and close to the cutoff frequency of the waveguide section. 
This configuration is also applied on the feeding waveguide WG2 of the horn antenna which is discussed in Section 2. By following similar steps as in the case of a straight waveguide, we obtained the results of the horn antenna after adding the tapered section at WG2 and extracting the parameters of this tapered section from the obtained results as shown in Figure 10. It can be noted that the reflection coefficient of Port 2 in this case is $0 \mathrm{~dB}$ where it is below cutoff frequency as shown in Figure 10(a) while it is less than $-10 \mathrm{~dB}$ above the cutoff frequency as shown in Figure 10(b).

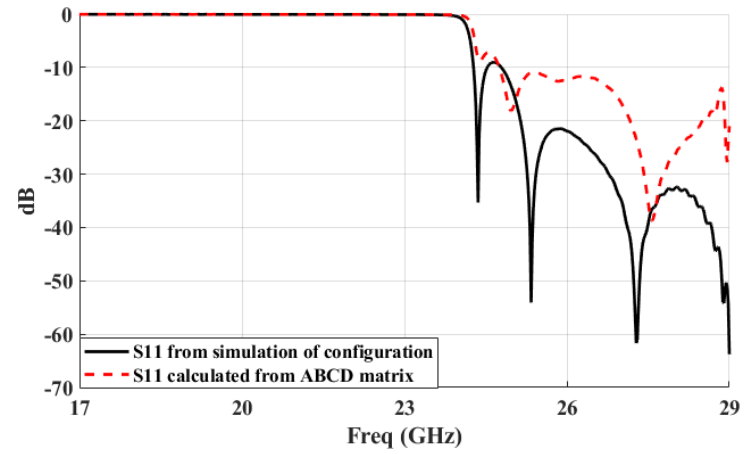

(a)

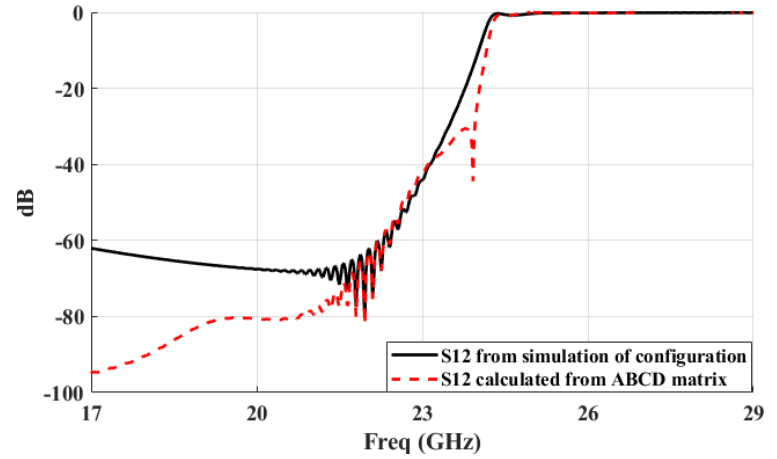

(b)

Figure 9. Coefficients (a) reflection and (b) transmission; (solid lines) with tapered sections, (dashed lines) without the tapered sections

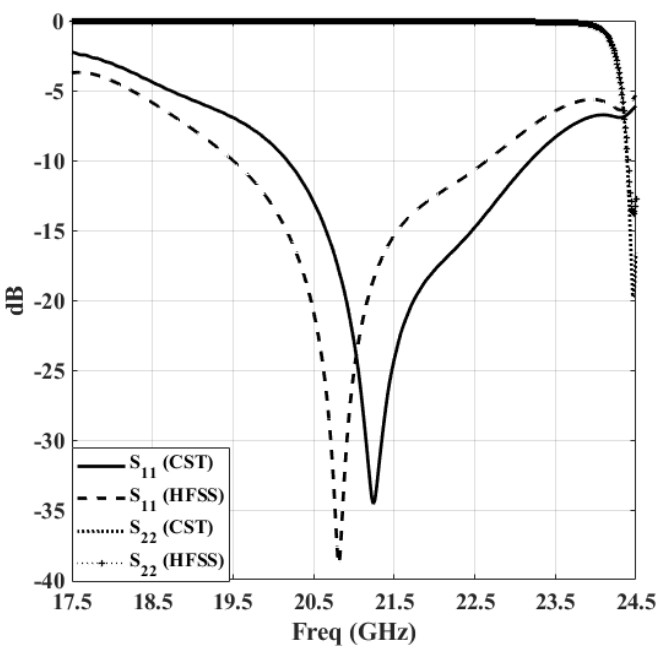

(a)

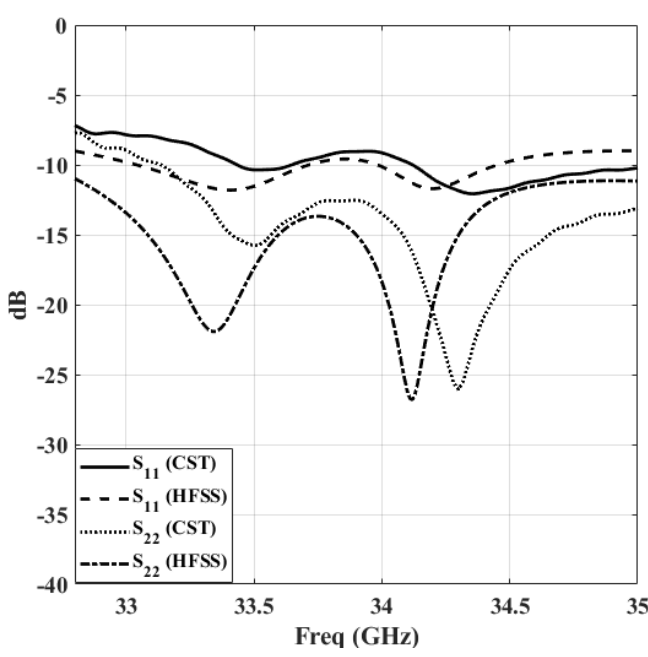

(b)

Figure 10. Simulated reflection coefficient of the conical horn antenna feed by two rectangular waveguide ports with TWG section at port 2` (a) Lower frequency band and (b) Upper frequency band

\section{CONCLUSION}

In this paper, we discussed a common problem in electromagnetic CAD tools like HFSS, CST and FEKO where the reflection coefficients of wave-ports close to and below their cutoff frequencies have unreasonable values. This problem is investigated in this paper on two examples; multiband horn antenna fed by two different rectangular waveguides and a straight waveguide section. To solve this problem, a waveguide tapered section is used as an excitation to the feeding waveguide where the feeding port of this tapered waveguide section has a cutoff frequency greater than the lowest frequency of interest in the simulation. The results of two tapered sections connected to each other are used to extract the parameters of a single tapered section. Then, this tapered section is added to the original problem under investigation to obtain the correct performance close to and below the cutoff frequency of the feeding waveguide. The parameters of the tapered section is then extracted from the results of the structure combined with the tapered section to obtain the results of the original structure separately. 


\section{REFERENCES}

[1] “Ansys HFSS|3D high frequency simulation software," Ansys. https://www.ansys.com/products/electronics/ansys-hfss (accessed Jan. 11, 2021).

[2] Dassault Systems, "CST Studio Suite 3D EM simulation and analysis software," BMC Public Health, 2020. https://www.3ds.com/products-services/simulia/products/cst-studio-suite/ (accessed Jan. 11, 2021).

[3] “Altair Feko Application.” ALTAIR. https://www.altair.com/feko-applications/ (accessed Jan. 11, 2021).

[4] M. Mujawar, T. Gunasekaran, and A. Rashid, "Design and analysis of X band pyramidal horn antenna using FEKO," in Proceedings of the 2021 1st International Conference on Advances in Electrical, Computing, Communications and Sustainable Technologies, ICAECT 2021, Feb. 2021, pp. 1-4, doi: 10.1109/ICAECT49130.2021.9392523.

[5] U. Jakobus, M. Bingle, M. Schoeman, J. J. Van Tonder, and F. Illenseer, "Tailoring FEKO for microwave problems," IEEE Microwave Magazine, vol. 9, no. 6, pp. 76-85, Dec. 2008, doi: 10.1109/MMM.2008.929557.

[6] W. O'Keefe Coburn, T. K. Anthony, and A. I. Zaghloul, "Open-ended waveguide radiation characteristics-Full-wave simulation versus analytical solutions," in 2010 IEEE International Symposium on Antennas and Propagation and CNC-USNC/URSI Radio Science Meeting - Leading the Wave, AP-S/URSI 2010, Jul. 2010, pp. 1-4, doi: 10.1109/APS.2010.5560987.

[7] A. Z. Elsherbeni, P. Nayeri, and C. J. Reddy, Antenna analysis and design using FEKO electromagnetic simulation software. Institution of Engineering and Technology, 2014.

[8] U. Nissanov and G. Singh, "MmWave/THz reconfigurable ultra-wideband (UWB) microstrip antenna," Progress In Electromagnetics Research C, vol. 111, pp. 207-224, 2021, doi: 10.2528/PIERC21012208.

[9] M. A. Al-Janabi and S. K. Kayhan, "Flexible vivaldi antenna based on a fractal design for Rf-energy harvesting," Progress In Electromagnetics Research M, vol. 97, pp. 177-188, 2020, doi: 10.2528/pierm20073003.

[10] K. H. Sayidmarie and K. M. Younus, "Analysis and design of two-slot antennas for wireless communication applications," Progress In Electromagnetics Research C, vol. 104, pp. 115-128, 2020, doi: 10.2528/pierc20050303.

[11] V. R. Kapure, P. P. Bhavarthe, and S. S. Rathod, "Tunable triple band-notched uwb antenna using single EBG and varactor diode," Progress In Electromagnetics Research C, vol. 110, pp. 181-195, 2021, doi: 10.2528/PIERC21012204.

[12] P. Martin, K. P. Ray, and N. N. S. S. R. K. Prasad, "Characterization study of mutual coupling between monopole antennas on finite ground plane at out of band resonant frequencies," Progress In Electromagnetics Research C, vol. 101, pp. 247-259, 2020, doi: 10.2528/PIERC20031604.

[13] H.-T. Chou, C.-W. Liu, H.-H. Chou, and W.-J. Liao, "Optimum horn antenna design based on an integration of HFSS commercial code and genetic algorithms for the feed application of reflector antennas," Applied Computational Electromagnetics Society Journal, vol. 25, no. 2, pp. 117-128, 2010.

[14] W. M. Hassan, K. M. Ibrahim, E. A. Abdallah, and A. M. Attiya, "Non-uniform feeding network for a dual circularly polarized $16 \times 16 \mathrm{Ku}$-band antenna array for on-move satellite communication," Applied Computational Electromagnetics Society Journal, vol. 36, no. 2, pp. 115-125, Mar. 2021, doi: 10.47037/2020.ACES.J.360201.

[15] B. Rachid, B. Bouhafs, and C. Mohammed, "ANSYS-HFSS Coupling matrix extraction with admittance parameters for microwave bandpass filter tuning," Nov. 2019, doi: 10.1109/ISPA48434.2019.8966930.

[16] C. Tian, Y. C. Jiao, G. Zhao, and H. Wang, "A wideband transmitarray using triple-layer elements combined with cross slots and double square rings," IEEE Antennas and Wireless Propagation Letters, vol. 16, pp. 1561-1564, 2017, doi: 10.1109/LAWP.2017.2651027.

[17] C. S. Prasad and A. Biswas, "Planar excitation of dielectric waveguide antenna for broadband and high-gain application," IEEE Antennas and Wireless Propagation Letters, vol. 16, pp. 1209-1212, 2017, doi: 10.1109/LAWP.2016.2628164.

[18] A. Taflove, S. C. Hagness, and M. Piket-May, "Computational electromagnetics: the finite-difference time-domain method," in The Electrical Engineering Handbook, Elsevier, 2005, pp. 629-670.

[19] R. F. Barrington, Field computation by moment methods. IEEE, 1993.

[20] P. P. Silvester and R. L. Ferrari, Finite Elements for Electrical Engineers. Cambridge University Press, 1996.

[21] D. M. Pozar, "Microwave Engineering, 4th Edition," John Wiley \&Sons, Inc, pp. 1-756, 2012.

[22] N. A. Aboserwal, C. A. Balanis, and C. R. Birtcher, "Conical horn: Gain and amplitude patterns," IEEE Transactions on Antennas and Propagation, vol. 61, no. 7, pp. 3427-3433, Jul. 2013, doi: 10.1109/TAP.2013.2256453.

[23] G. Abhignya, B. Yogita, C. Abhinay, B. Balaji, and M. B. R. Murthy, "Design, fabrication and testing of pyramidal horn antenna," International Journal of Engineering and Applied Sciences, vol. 2, no. 4, 2015.

[24] B. Edwards, A. Alù, M. E. Young, M. Silveirinha, and N. Engheta, "Experimental verification of epsilon-near-zero metamaterial coupling and energy squeezing using a microwave waveguide," Physical Review Letters, vol. 100, no. 3, Jan. 2008, doi: 10.1103/PhysRevLett.100.033903.

[25] T. Tang, J. Li, Y. Zhang, C. Li, and L. Luo, "Spin Hall effect of transmitted light in a three-layer waveguide with lossy epsilonnear-zero metamaterial," Optics Express, vol. 24, no. 24, p. 28113, Nov. 2016, doi: 10.1364/oe.24.028113.

[26] S. Dwari, A. Chakraborty, and S. Sanyal, "Analysis of linear tapered waveguide by two approaches," Progress in Electromagnetics Research, vol. 64, pp. 219-238, 2006, doi: 10.2528/PIER06071902.

[27] J. A. B. Faria, "Nonuniform transmission-line structures: internal and external propagation parameters," Electrical Engineering, vol. 87, no. 1, pp. 19-22, Jan. 2005, doi: 10.1007/s00202-003-0217-z.

\section{BIOGRAPHIES OF AUTHORS}

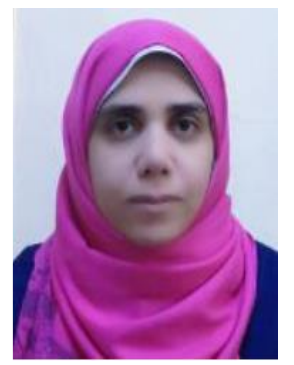

Eman Mohmaed Eldesouki (iD) SC P Electronics and Electrical Communications, Faculty of Engineering, Benha University in 2003, at Cairo University in 2009, and at Ain Shams University in 2018, respectively. She joined Electronics Research Institute as a Researcher Assistant in 2005. Her research interests include Antennas, Electromagnetic wave scattering from rough surfaces, Wave propagations, Synthetic aperture radars (SAR), Polarimetric radar imaging, filter design, microwave measurement techniques and Numerical techniques in electromagnetics. Email: eman @eri.sci.eg. 


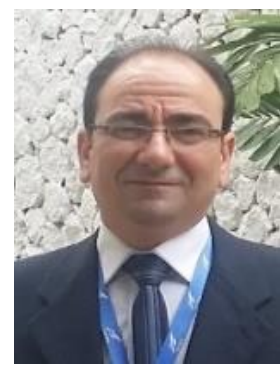

Khalid M. Ibrahim (D) SC SC Electronics and Electrical Communications, Faculty of Engineering, Al-Azhar University at 2000,2007, and 2014 respectively. He joined Electronics Research Institute as a Researcher Assistant in 2001. His research interests include Antennas, Electromagnetic waves, Antennas and wave propagations, Ground penetrating radars, Antenna and Microwave measurement techniques, Numerical techniques in electromagnetics. Email: khaledmus@gmail.com.

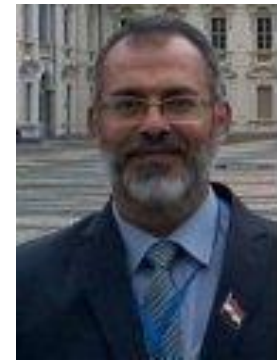

Ahmed M. Attiya (D) SC P Electronics and Electrical Communications, Faculty of Engineering, Cairo University in 1996 and 2001 respectively. He joined Electronics Research Institute as a Researcher Assistant in 1991. From 2002 to 2004 he was a Postdoc in Bradley Department of Electrical and Computer Engineering at Virginia Tech. From 2004 to 2005 he was a Visiting Scholar in Electrical Engineering Dept. in University of Mississippi. From 2008 to 2012 he was a Visiting Teaching Member in King Saud University. He is currently Full Professor and the Head of Microwave Engineering Dept. in Electronics Research Institute. His research interests include Electromagnetic waves, antennas and wave propagations, microwave passive circuits and systems, microstrip and planar circuits and antennas, antenna measurement techniques, microwave measurement techniques, UWB and short pulse signals, numerical techniques in electromagnetics, analytical techniques in electromagnetics, periodic structures, artificial electromagnetic materials, nanotechnology, carbon nanotubes, graphene, and plasmonics. Email: ahmed_attiya@ @otmail.com. 